INPLASY

PROTOCOL

To cite: Li et al. The safety and efficacy of flow diverter versus conventional endovascular treatment in intracranial aneurysm: a meta-analysis of the real-world cohort studies over last ten years. Inplasy protocol 2021100061. doi: 10.37766/inplasy2021.10.0061

Received: 17 October 2021

Published: 17 October 2021

Corresponding author: Fenghua Chen

xyswcfh@csu.edu.cn

Author Affiliation:

Xiangya hospital, Central

South University.

Support: None.

Review Stage at time of this submission: Piloting of the study selection process.

Conflicts of interest: None declared.

\section{The safety and efficacy of flow diverter versus conventional endovascular treatment in intracranial aneurysm: a meta-analysis of the real-world cohort studies over last ten years}

\author{
Li, SF1; Tao, WG2; Zeng, CD3\% Yan, LC4; Chen, FH5.
}

Review question / Objective: Did adult patients with intracranial aneurysms (patient population) who underwent flow diverter (FD, Intervention) have better clinical outcomes, higher rates of aneurysm occlusion, and lower rates of mortality and procedure related complications (outcomes) when compared with patients who received the conventional endovascular treatments (CEV, control) from January 2010 to December 2020?

Eligibility criteria: The inclusion criteria are: 1. directly comparison of FD and conventional endovascular treatment, including coiling alone, stent alone, stent-assisted coiling, and balloon-assisted coiling; 2 . patients $\geq 18$ years with intracranial aneurysms; 3 . detailed follow up angiographic and clinical outcomes; 4 . the number of patients in two groups at least 10 patients. The exclusion criteria are:1. less than 10 participants in either group; 2. without the report of outcome variables; 3. studies primarily focus on children patients; 4. studies primarily focus on one interventional tool.

INPLASY registration number: This protocol was registered with the International Platform of Registered Systematic Review and Meta-Analysis Protocols (INPLASY) on 17 October 2021 and was last updated on 17 October 2021 (registration number INPLASY2021100061).

\section{INTRODUCTION}

Review question / Objective: Did adult patients with intracranial aneurysms (patient population) who underwent flow diverter (FD, Intervention) have better clinical outcomes, higher rates of aneurysm occlusion, and lower rates of mortality and procedure related complications (outcomes) when compared with patients who received the conventional endovascular treatments (CEV, control) from January 2010 to December 2020?

Condition being studied: Rapid technological advances in endovascular 
treatment have been transforming the modality of intracranial aneurysms (IAs) treatment in the past years. The Guglielmi detachable coil, introduced in the early 1990s, revolutionarily provided an alternative to traditional surgical clipping in treatment of IAs. After that, the reconstructive techniques such as balloon assisted coiling and stent assisted coiling (SAC) were initially utilized. Most recently, low-profile visualized intraluminal support (LVIS) as a self-expandable, recyclable, and braided stent were also widely adopted to clinical practice s. Compared with these standard and conventional stent methods, flow diversion (FDs) like pipeline embolization device (PED) approved by the Food and Drug Administration (FDA) in 2011 have a larger mental coverage and present its broader indications for the treatment of complex aneurysms such as large and giant ICA aneurysms and fusiform, dissecting, and blood blister-like aneurysms . However, the high rate of aneurysms rupture, procedural mortality, and morbidity after placement of FDs also raised much concerns. It is crucial to assess the risk-benefit ratio for treatment of flow diverters (FDs) by comparing it with the conventional endovascular treatments.

\section{METHODS}

Search strategy: Search strategies: 1. For Pubmed, the search used was:

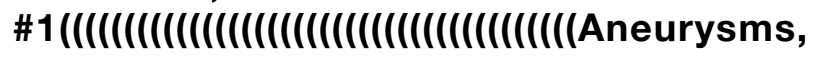
Intracranial[Title/Abstract]) OR (Intracranial Aneurysms[Title/Abstract])) OR (Aneurysm, Intracranial[Title/Abstract])) OR (Aneurysm, Anterior Communicating Artery[Title/ Abstract])) OR (Anterior Communicating Artery Aneurysm[Title/Abstract])) OR (Aneurysm, Basilar Artery[Title/Abstract])) OR (Aneurysms, Basilar Artery[Title/ Abstract])) OR (Artery Aneurysm, Basilar[Title/Abstract])) OR (Artery Aneurysms, Basilar[Title/Abstract])) OR (Basilar Artery Aneurysms[Title/Abstract])) OR (Basilar Artery Aneurysm[Title/ Abstract])) OR (Aneurysm, Middle Cerebral Artery[Title/Abstract])) OR (Middle Cerebral Artery Aneurysm[Title/Abstract])) OR (Aneurysm, Posterior Cerebral Artery[Title/
Abstract])) OR (Posterior Cerebral Artery Aneurysm[Title/Abstract])) OR (Berry Aneurysm[Title/Abstract])) OR (Aneurysm, Berry[Title/Abstract])) OR (Aneurysms, Berry[Title/Abstract])) OR (Aneurysm, Brain[Title/Abstract])) OR (Aneurysms, Brain[Title/Abstract])) OR (Brain Aneurysms[Title/Abstract])) OR (Cerebral Aneurysm[Title/Abstract])) OR (Aneurysms, Cerebral[Title/Abstract])) OR (Aneurysm, Cerebral[Title/Abstract])) OR (Giant Intracranial Aneurysm[Title/Abstract])) OR (Aneurysm, Giant Intracranial[Title/ Abstract])) OR (Aneurysms, Giant Intracranial[Title/Abstract])) OR (Giant Intracranial Aneurysms[Title/Abstract])) OR (Intracranial Aneurysm, Giant[Title/ Abstract])) OR (Intracranial Aneurysms, Giant[Title/Abstract])) OR (Intracranial Aneurysms, Giant[Title/Abstract])) OR (Mycotic Aneurysm, Intracranial[Title/ Abstract])) OR (Aneurysm, Intracranial Mycotic[Title/Abstract])) OR (Aneurysms, Intracranial Mycotic[Title/Abstract])) OR (Intracranial Mycotic Aneurysm[Title/ Abstract])) OR (Mycotic Aneurysms, Intracranial[Title/Abstract])) OR (Aneurysm, Anterior Cerebral Artery[Title/Abstract])) OR (Anterior Cerebral Artery Aneurysm[Title/Abstract])) OR (Aneurysm, Posterior Communicating Artery[Title/ Abstract])) OR (Posterior Communicating Artery Aneurysm[Title/Abstract])) OR ("Intracranial Aneurysm"[Mesh]) \#2 ()(()((()flower diverter[Title/Abstract]) OR (flow diversion[Title/Abstract])) OR (flow diverter embolization[Title/Abstract])) OR (pipeline embolization[Title/Abstract])) OR (pipeline flex[Title/Abstract])) OR (pipeline device[Title/Abstract])) OR (flow diverting stent[Title/Abstract])) OR (Surpass Streamline [Title/Abstract])) OR (Tubridge[Title/Abstract])) OR (Surpass Streamline[Title/Abstract])) OR (p64 (device)[Title/Abstract]) \#3 \#1 AND \#2 | 2. For Embase, the search used was: \#1 'intracranial aneurysm'/exp \#2 'internal carotid artery aneurysm'/exp \#3 \#1 OR \#2 \#4 'flow diverter'/exp OR ' pipeline embolization device'/exp \#5 \#3 AND \#4 3. For Cochrane, the search used was: \#1 MeSH descriptor: [Intracranial Aneurysm] explode all trees \#2 (aneurysms, intracranial):ti,ab,kw OR (anterior 
communicating artery aneurysm):ti,ab,kw OR (aneurysm, anterior communicating artery):ti,ab,kw OR (aneurysm, basilar artery):ti,ab,kw OR (basilar artery aneurysm):ti,ab,kw OR (middle cerebral artery aneurysm):ti,ab,kw OR (aneurysm, middle cerebral artery):ti, ab,kw OR (posterior cerebral artery aneurysm):ti,ab,kw OR (aneurysm, posterior cerebral artery):ti,ab,kw OR (posterior communicating artery aneurysm):ti,ab,kw OR (aneurysm, posterior communicating artery):ti,ab,kw OR (brain aneurysm):ti,ab,kw OR (cerebral aneurysm) \#3 \#1 OR \#2 | \#4 (flow diverter): ti, ab, kw OR (flow diversion):ti,ab,kw OR (flow diverting stent):ti,ab,kw OR (P64(device)):ti,ab,kw OR (Surpass Streamline):ti,ab,kw OR (Tubridge):ti,ab,kw OR (pipeline embolization):ti,ab,kw OR (pipeline flex):ti,ab,kw OR (pipeline device) | \#5 \#3 AND \#4.

Participant or population: The inclusion criteria are:1. patients $\geq 18$ years with intracranial aneurysms;2. detailed follow up angiographic and clinical outcomes; 3 . the number of patients in two groups at least 10 patients.The exclusion criteria are: 1. less than 10 participants in either group;2. without the report of outcome variables; 3 . studies primarily focus on children patients.

Intervention: The inclusion criteria are:Directly comparison of FD and conventional endovascular treatment, including coiling alone, stent alone, stentassisted coiling, and balloon-assisted coiling;The exclusion criteria are:Studies primarily focus on one interventional tool.

Comparator: Flow diverter versus conventional endovascular treatments,, including coiling alone, stent alone, stentassisted coiling, and balloon-assisted coiling in intracranial aneurysmveruse.

Study designs to be included: The inclusion criteria are:1. directly comparison of FD and conventional endovascular treatment, including coiling alone, stent alone, stentassisted coiling, and balloon-assisted coiling;2. patients $\geq 18$ years with intracranial aneurysms;3. detailed follow up angiographic and clinical outcomes;4. the number of patients in two groups at least 10 patients.The exclusion criteria are:1. less than 10 participants in either group;2. without the report of outcome variables;3. studies primarily focus on children patients;4. studies primarily focus on one interventional tool.

Eligibility criteria: The inclusion criteria are: 1. directly comparison of FD and conventional endovascular treatment, including coiling alone, stent alone, stentassisted coiling, and balloon-assisted coiling; 2 . patients $\geq 18$ years with intracranial aneurysms; 3 . detailed follow up angiographic and clinical outcomes; 4. the number of patients in two groups at least 10 patients. The exclusion criteria are: 1. less than $\mathbf{1 0}$ participants in either group; 2. without the report of outcome variables; 3. studies primarily focus on children patients; 4. studies primarily focus on one interventional tool.

Information sources: Our searches are from PubMed, EMBASE, and Cochrane database.

Main outcome(s): 1. procedure-related complications 2 . immediated occlusion rate 3. long-term occlusion rate $4 . \mathrm{mRS}$ at follow-up.

Additional outcome(s): 1. baseline data, such as the age, gender, hypertension, and aneurysm sizes 2 . the recurrence and retreatment rate in the long-term.

Data management: References generated from these searches were imported into the reference manager EndNote X9 (Thompson Reuters, Philadelphia, PA), and two authors (Zeng and Tao) systematically screened out the references independently according to the inclusion criteria. The discrepancies were resolved after discussing with the third author (Li).Additionally, studies were only included if published in English and in an original article. Review articles, abstract, case reports, systematic reviews and meta- 
analyses, letters to the editor, reviews, editorials, commentaries, studies on animal models, and basic science studies were not considered.

Quality assessment / Risk of bias analysis: $A$ review and the data extraction of all included studies were performed by 3 authors (Zeng, Tao, and Li) independently. The disagreements were also resolved by consensus in meetings with all authors. Quality of included studies was assessed using the Newcastle-Ottawa Scale (NOS) for cohort studies. This scale rates studies based on three major aspects: selection, comparability, and the ascertainment of outcome of interest. We identified highquality choices by adding a star to the questions in each aspect. The more stars allocated to a study, the better the quality it was. We included all eligible studies regardless of their assessed quality.

Strategy of data synthesis: This metaanalysis was conducted in $R$ software (version R-4.1.0) with the "meta" package. Dichotomous data from included studies were used to generate odds ratios (ORs) and continuous data were used for standard mean difference (SMD) with 95\% confidence intervals (Cls) by DerSimonian and Laird models with inverse variance weighting method. A random -effect model was used if the outcome with high heterogeneity assessed and noted with $12>$ $50 \%$, otherwise the fixed-effect model applied. The sources of heterogeneity were explored by subgroup analysis, metaregression, and sensitivity analysis by sequential exclusion of 1 study at a time. A funnel plot with Egger's regression test was depicted for evaluating the publication bias. Statistical significance was identified with a $P<0.05$.

Subgroup analysis: The aneurysm size and study type (that is, whether this study was matched by propensity score matching or other method) will be used as the subgroup analysis.

Sensitivity analysis: Sensitivity analysis will be conducted by sequential exclusion of 1 study at a time.
Language: English.

Country(ies) involved: China.

Other relevant information: None.

Keywords: intracranial aneurysm; flow diverting; endovascular treatment; metaanalysis.

Dissemination plans: We wish this metaanalysis will be published in a high-quality journal.

Contributions of each author:

Author 1 - Shifu Li - The author drafted the manuscript.

Email: shifuli@csu.edu.cn

Author 2 - Wengui Tao - The author provided statistical expertise.

Email: t361021775@163.com

Author 3 - Chudai Zeng - The author contributed to the development of the selection criteria, and the risk of bias assessment strategy.

Email: engchudai@csu.edu.cn

Author 4 - Langchao Yan - The author collected the data and reviewed the manuscript.

Email: ylc19950115@163.com

Author 5 - Fenghua Chen - The author read, provided feedback and approved the final manuscript.

Email: xyswcfh@csu.edu.cn 